\title{
THE CENTER OF A JORDAN RING 1
}

\section{N. JACOBSON}

If $\mathfrak{A}$ is an arbitrary associative ring we can symmetrize and antisymmetrize the multiplication defined in $\mathfrak{A}$ to obtain two nonassociative rings. We set

$$
\{a b\}=a b+b a, \quad[a b]=a b-b a
$$

and call the former the Jordan product and the latter the commutator or Lie product of $a$ and $b$. If we use $\{a b\}$ as product in place of the originally defined $a b$ we obtain the Jordan ring $\mathfrak{A}_{j}$ determined by $\mathfrak{A}$. Similarly the Lie ring $\mathfrak{A}_{l}$ is obtained by using $[a b]$ in place of $a b$. Naturally if $\mathfrak{A}$ has characteristic 2 then $\mathfrak{A}_{j}=\mathfrak{A}_{l}$. It is customary to exclude this case from consideration but in most of our discussion we shall not find it necessary to do so. Clearly $\{a b\}=\{b a\},[a b]$ $=-[b a]$. Also we recall the following well known identity of Jacobi's:

$$
[[a b] c]+[[b c] a]+[[c a] b]=0 .
$$

If $\Re$ is any non-associative ring one defines the center of $\Re$ to be the totality of elements $c$ that commute,

$$
c \cdot a=a \cdot c,
$$

and associate,

$$
\begin{gathered}
(a \cdot b) \cdot c=a \cdot(b \cdot c), \quad(a \cdot c) \cdot b=a \cdot(c \cdot b), \\
(c \cdot a) \cdot b=c \cdot(a \cdot b),
\end{gathered}
$$

with all $a, b$ in $\Re .^{2}$ It is known that the center is a subring of $\Re$. Clearly this subring is associative. It is also known that the center of a simple ring is either 0 or a field. It is easy to see that the middle condition in (4) is a consequence of (3) and the other conditions in (4). Also it is clear that if $\Re$ is commutative then the first condition of (4) characterizes the center.

We consider now the centers $\mathfrak{C}_{j}$ and $\mathfrak{C}_{l}$ respectively of $\mathfrak{A}_{j}$ and $\mathfrak{A}_{l}$.

Received by the editors May 6, 1947, and, in revised form, July 3, 1947.

${ }^{1} \mathrm{I}$ am indebted to A. H. Clifford for a number of valuable conversations on the subject of this note.

${ }^{2}$ See Jacobson, Structure theory of simple rings without finiteness assumptions, Trans. Amer. Math. Soc. vol. 57 (1945) p. 239, or T. Nakayama, Über einfache distributive Systeme unendlicher Ränge, Proc. Imp. Acad. Tokyo vol. 20 (1944) p. 62 for this definition and for the results quoted in this paragraph. 
First let $c \in \mathfrak{C}_{l}$. Then by (1) and (3), $2[c a]=0$. By (4) and Jacobi's identity, $[[c a] b]=0$. Hence

$$
2[c a]=0, \quad[[c a] b]=0
$$

holds. It is also easy to see that these conditions are sufficient that $c \in \mathfrak{C}_{l}$.

Next let $c \in \mathfrak{C}_{j}$. We introduce the Jordan associator

$$
A(a, b, c)=\{\{a b\} c\}-\{a\{b c\}\}
$$

and we can verify that

$$
A(a, b, c)=[[c a] b]
$$

Hence

$$
[[c a] b]=0
$$

is a necessary and sufficient condition that $c \in \mathbb{C}_{j}$. Thus we see that $\mathfrak{E}_{l} \subseteq \mathfrak{C}_{j}$. If we denote the center of $\mathfrak{A}$ by $\mathfrak{E}, c \in \mathfrak{C}$ if and only if $[c a]=0$. Hence $\mathbb{E} \subseteq \mathbb{E}_{l} \subseteq \mathbb{E}_{j}$.

Let $c \in \mathfrak{C}_{j}$ and $a, b, d$ be arbitrary in $\mathfrak{A}$. Then $[[c, a b] d]=0$. Since

$$
[c, a b]=[c a] b+a[c b]
$$

we obtain

$$
[c a][b d]+[a d][c b]=0 .
$$

This simple relation has a number of interesting consequences. In the first place if we set $d=c^{\prime}$, a second element in $\mathfrak{\mho}_{j}$, we obtain $[c a]\left[b c^{\prime}\right]$ $+\left[a c^{\prime}\right][c b]=0$. If we use the fact that $[c b] \in \mathbb{C}$ this reads

$$
[c a]\left[c^{\prime} b\right]+[c b]\left[c^{\prime} a\right]=0 .
$$

It is easy to see that this implies

$$
\left[\left[c c^{\prime}, a\right] b\right]=0 .
$$

Thus $c c^{\prime} \in \mathfrak{C}_{j}$. It is clear also that if $2[c a]=0$ and $2\left[c^{\prime} a\right]=0$ then $2\left[c c^{\prime}, a\right]=0$. Hence we have the following theorem.

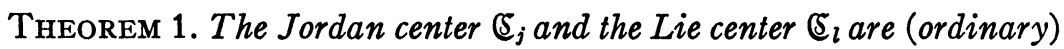
subrings of $\mathfrak{A}$.

If we specialize $d=a$ and $b=c$ successively in (8) we obtain

$$
[c a][b a]=0, \quad[c a][c d]=0 .
$$

In particular 


$$
[c a]^{2}=0 .
$$

This implies the following theorem.

THEOREM 2. If $\mathfrak{A}$ is an associative ring whose center (S) contains no nilpotent elements not equal to 0 then the Jordan center $\mathfrak{E}_{j}$ coincides with c.

For if $c \in \mathfrak{C}_{j},[c a]^{2}=0$ for any $a \in \mathfrak{A}$. Since $[c a] \in \mathbb{C}$ this implies that $[c a]=0$. Hence $c \in \mathfrak{C}$.

It is easy to see that if a ring contains a nilpotent element in its center then it contains a nilpotent two-sided ideal. Hence we have the following corollary.

COROLLARY. If $\mathfrak{A}$ is a ring that has no nilpotent two-sided ideals then its Jordan center $\mathfrak{S}_{j}$ coincides with the ordinary center.

It is clear from Jacobi's identity that if $c \in \mathfrak{C}_{j}$ then $[[a b] c]=0$ for all $a, b$. Thus $c$ commutes with every commutator. Clearly the elements that have this property form a subring $\mathfrak{B}$ of $\mathfrak{A}$. It will be shown in a forthcoming paper by Kaplansky that if $\mathfrak{A}$ is a semi-simple ring then $\mathfrak{B}=\mathfrak{C} .{ }^{3}$ Thus Kaplansky's result has both a stronger hypothesis and a stronger conclusion than our Theorem 2. The following examples will serve to illustrate these results.

EXAMPLE 1. Let $\mathfrak{A}$ be the ring of triangular matrices

$$
\left(\begin{array}{lll}
\alpha_{11} & & * \\
& \alpha_{22} & \\
& & \\
0 & & \alpha_{n n}
\end{array}\right)
$$

with elements in a field $\Phi$. It is easy to see that $\mathfrak{C}$ is the set of scalar matrices. Hence $\mathfrak{E}_{j}=\mathfrak{c}$. On the other hand it can be seen that $\mathfrak{B}$ is the set of matrices

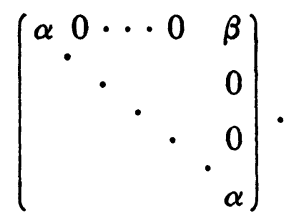

EXAMPLE 2. Let $\mathfrak{A}$ be the set of triangular matrices for which $\alpha_{11}=\alpha_{22}=\cdots=\alpha_{n n}=\alpha$. Here $\mathbb{E}$ is the set of matrices of the form

${ }^{3}$ Added in proof. This paper has now appeared: Semi-automorphisms of rings, Duke Math. J. vol. 14 (1947).pp. 521-527. 
(14) and $\mathscr{B}$ is the set of matrices of the form

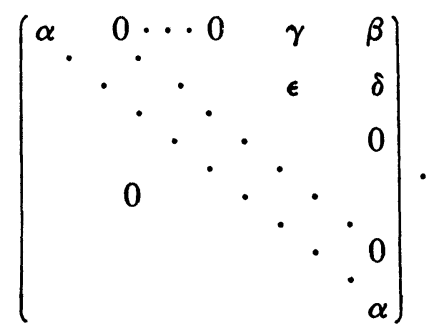

Finally $\mathfrak{C}_{j}$ is the subset of $\mathfrak{B}$ of the matrices of the form (15) in which $\epsilon=0$.

We derive next another property of the element $c$ in $\mathfrak{C}_{j}$. If $a, b, d$ are arbitrary in $\mathfrak{A}$,

$$
\begin{aligned}
{[[c a, b] d] } & =[(a[c b]+c[a b]), d] \\
& =[a d][c b]+[c d][a b]+c[[a b] d] .
\end{aligned}
$$

If we interchange $a$ and $d$ in (8) we see that

$$
[a d][c b]+[c d][a b]=0 .
$$

Hence

$$
[[c a, b] d]=c[[a b] d] .
$$

This implies also that $[[a, c b] d]=c[[a b] d]$. By Jacobi's identity we obtain finally $[[a b] c d]=c[[a b] d]$. Thus

$$
c[[a b] d]=[[c a, b] d]=[[a, c b] d]=[[a b] c d] .
$$

This can also be written in the following form in terms of the associator $A(b, d, a)=[[a b] d]$ :

$$
c A(b, d, a)=A(b, d, c a)=A(c b, d, a)=A(b, c d, a) .
$$

In the remainder of this note we consider rings $\mathfrak{U}$ that are subrings of a Jordan ring of the form $\mathfrak{A}_{j}$. By the enveloping (associative) ring of $\mathfrak{U}$ in $\mathfrak{A}$ we mean the subring of $\mathfrak{A}$ generated by $\mathfrak{U}$. If $\mathfrak{E}$ is the enveloping ring of $\mathfrak{U}$ clearly $\mathfrak{U}$ is also a subring of the Jordan ring $\mathfrak{E}_{j}$. Hence we can suppose that $\mathbb{E}=\mathfrak{A}$.

If $c$ is in the Jordan center $\mathfrak{E}_{j}(\mathfrak{U})$ of $\mathfrak{u}$ then $[[c a] b]=0$ holds for all $a, b \in \mathfrak{U}$. Since $\mathfrak{U}$ is a Jordan ring

$$
[[c,\{a b\}] d]=0
$$

for all $a, b, d$ in $\mathfrak{u}$. Also we have $[c[a b]]=0$ so that $[[c,[a b]] d]=0$. Hence by addition 


$$
2[[c, a b] d]=0 .
$$

We shall assume now that the ring $\mathfrak{A}$ contains no element that has order two in the additive group. Then by (17), $[[c, a b] d]=0$ for all $a, b, d$ in $\mathfrak{u}$. As before this leads to the relation (8) and to its consequence $[c a]^{2}=0$. On the other hand since $[[c a] b]=0,[c a]$ commutes with every $b$ in $\mathfrak{U}$. Hence it commutes with every $b$ in the enveloping ring $\mathfrak{A}$. Thus $[c a]$ is in the center $\mathfrak{E}$ of $\mathfrak{A}$. This shows that if some $[c a] \neq 0$ then the center of $\mathfrak{A}$ possesses nilpotent elements. Hence we have the following theorem.

THEOREM 3. Let $\mathfrak{A}$ be an associative ring that has no elements of order 2 and that has no nilpotent elements $(\neq 0)$ in its center. Let $\mathfrak{U}$ be a subring of the Jordan ring $\mathfrak{A}_{j}$ such that the enveloping ring of $\mathfrak{u}$ is $\mathfrak{A}$. Then the Jordan center $\mathfrak{E}_{j}(\mathfrak{U})$ coincides with the totality of elements $c$ of $\mathfrak{U}$ that commute with every $a$ in $\mathfrak{U}$ (or in $\mathfrak{2})$.

A. A. Albert has recently studied the structure of the subalgebras $\mathfrak{U}$ of a Jordan algebra $\mathfrak{A}_{j}, \mathfrak{A}$ a matrix algebra $\Phi_{n}$ over a field $\Phi .{ }^{4}$ In the course of this study he has defined the center of $\mathfrak{u}$ to be the totality of elements $c$ such that $[c a]=0$ for all $a$ in $\mathfrak{u}$. We shall show in an example that Albert's definition is unsatisfactory for arbitrary Jordan algebras since it is not invariant under isomorphism. On the other hand it will also be shown that in the case of simple algebras over a field of characteristic 0 -and this case is the only one for which Albert uses his definition-Albert's center coincides with the Jordan center as defined in the present paper. We remark that Theorems 2 and 3 give other cases for which this identity holds. In all of these cases Albert's definition can not lead to any difficulties. We consider now the following example.

EXAmple 3. Let $\mathfrak{A}$ be the algebra over $\Phi$ that has the basis $z_{1}, z_{2}$ such that $z_{i} z_{j}=0$. The Jordan ring $\mathfrak{A}_{j}$ has the basis $z_{1}, z_{2}$ with the multiplication table

$$
\left\{z_{1} z_{1}\right\}=0, \quad\left\{z_{1} z_{2}\right\}=0, \quad\left\{z_{2} z_{2}\right\}=0 .
$$

Let $\mathfrak{B}$ be the algebra over $\Phi$ that has the basis $Z_{1}, Z_{2}, Z_{3}$ with the multiplication table

$$
\begin{array}{cc}
Z_{1}^{2}=Z_{2}^{2}=Z_{3}^{2}=0, \quad Z_{1} Z_{2}=-Z_{2} Z_{1}=Z_{3}, \quad Z_{2} Z_{3}=Z_{3} Z_{2}=0, \\
Z_{3} Z_{1}=Z_{1} Z_{3}=0 .
\end{array}
$$

Since any product of three $Z$ 's is $0, \mathscr{B}$ is an associative algebra. We

4 On Jordan algebras of linear transformations, Trans. Amer. Math. Soc. vol. 59 (1946) p. 540. 
have the Jordan multiplication table

$$
\left\{Z_{1} Z_{1}\right\}=0, \quad\left\{Z_{1} Z_{2}\right\}=0, \quad\left\{Z_{2} Z_{2}\right\}=0 .
$$

Hence $\mathfrak{U}=\left(Z_{1}, Z_{2}\right)$ is a subalgebra of $\mathfrak{B}_{j}$ isomorphic to $\mathfrak{A}_{j}$. The totality of elements of $\mathfrak{A}_{j}$ that commute with all the elements of $\mathfrak{A}_{j}$ is $\mathfrak{A}_{j}$ itself. However, the elements $Z_{1}$ and $Z_{2}$ do not commute with all the elements of $\mathfrak{u}$. Evidently the Jordan center of $\mathfrak{A}_{j} \mathfrak{i s}_{\mathfrak{A}} \mathfrak{H}_{j}$.

We shall now consider the Jordan center of any simple Jordan algebra with a finite basis over a field of characteristic 0 . At first we consider any associative algebra $\mathfrak{A}$ over a field $\Phi$. As before let $\mathfrak{A}_{j}$ denote the Jordan algebra obtained from $\mathfrak{A}$ by using the operations $a+b, \alpha a$ for $\alpha$ in $\Phi$ and $\{a b\}=a b+b a$. If $\mathfrak{U}$ is a subalgebra of $\mathfrak{A}_{j}$ it is clear that the center $\mathfrak{E}_{j}(\mathfrak{u})$ is a subalgebra. Let $c \in \mathfrak{C}_{j}(\mathfrak{U})$ and let $a$ be any algebraic element of $\mathfrak{u}$. Then there exists a polynomial $\phi(\lambda) \neq 0$ in $\Phi[\lambda]$ such that $\phi(a)=0$. Since $[c a]$ commutes with $a,\left[c, a^{k}\right]=k a^{k-1}[c a]$. Hence

$$
0=[c, \phi(a)]=\phi^{\prime}(a)[c a]
$$

where $\phi^{\prime}(\lambda)$ is the derivative of the polynomial $\phi(\lambda)$. We assume next that $c$ is algebraic and that $a$ is arbitrary. Then a similar argument shows that if $\psi(\lambda)$ is a polynomial not equal to 0 such that $\psi(c)=0$ then

$$
\psi^{\prime}(c)[c a]=0 .
$$

We suppose now that $\mathfrak{U}=\Phi_{n}, \Phi$ of characteristic 0 . Let $\mathfrak{U}$ be a simple subalgebra of $\mathfrak{A}_{j}$. Then it has been shown by Albert that $\mathfrak{U}$ has an identity $e .{ }^{\mathfrak{b}}$ Evidently $e$ is in the Jordan center $\mathfrak{E}_{j}(\mathfrak{U})$. Hence $\mathfrak{E}_{j}(\mathfrak{U}) \neq 0$ and it follows that $\mathfrak{E}_{j}(\mathfrak{U})$ is a field. Since $e a+a e=a, e^{2}=e / 2$. If we set $e^{\prime}=2 e$ then $\left(e^{\prime}\right)^{2}=e^{\prime}$ and $\left(e^{\prime} a+a e^{\prime}\right) / 2=a$. Hence

$$
e^{\prime} a / 4+e^{\prime} a e^{\prime} / 2+a e^{\prime} / 4=\left(e^{\prime} a+a e^{\prime}\right) / 2
$$

and

$$
a=\left(e^{\prime} a+a e^{\prime}\right) / 2=e^{\prime} a e^{\prime} .
$$

It follows that $e^{\prime} a=a=a e^{\prime}$. Thus $e^{\prime}$ acts as an identity for all the elements of $\mathfrak{u}$ and therefore for all the elements of the enveloping algebra $\mathfrak{E}$ of $\mathfrak{u}$.

Suppose now that $c \in \mathfrak{C}_{j}(\mathfrak{U})$ and that $\mu(\lambda)$ is the minimum polynomial of $c$ regarded as an element of $\mathfrak{E}_{j}(\mathfrak{U})$. Then if $\mu(\lambda)=\lambda^{m}$

' Albert (loc. cit., footnote 3) uses the multiplication $a \cdot b=\{a b\} / 2$. Clearly an algebra is simple relative to the dot multiplication if and only if it is simple relative to \{\} . Moreover, if $e^{\prime}$ is an identity relative to $\cdot$, then $e=e^{\prime} / 2$ is one relative to \{\} . 
$+\gamma_{1} \lambda^{m-1}+\cdots+\gamma_{m}$,

$$
\{c\}^{m}+\gamma_{1}\{c\}^{m-1}+\cdots+\gamma_{m-1} c+\gamma_{m} e=0
$$

where $\{c\}^{r}$ is defined inductively by $\{c\}^{r}=\left\{\{c\}^{r-1}, c\right\}$. Since $\{c\}^{r}=2^{r-1} c^{r},(20)$ yields

$$
c^{m}+\frac{\gamma_{1}}{2} c^{m-1}+\cdots+\frac{\gamma_{m-1}}{2^{m-1}} c+\frac{\gamma_{m}}{2^{m}} e^{\prime}=0
$$

Thus if $\psi(\lambda)=2^{-m} \mu(2 \lambda)$ then $\psi(c)=0$. Since $\mathfrak{C}_{j}(\mathfrak{U})$ is a separable field, $\mu^{\prime}(\lambda)$ is prime to $\mu(\lambda)$. Hence $\psi^{\prime}(\lambda)$ is prime to $\psi(\lambda)$. It follows that

$$
\psi^{\prime}(c)=m c^{m-1}+(m-1) \frac{\gamma_{1}}{2} c^{m-2}+\cdots+\frac{\gamma_{m-1}}{2^{m-1}} e^{\prime}
$$

has an ordinary inverse relative to $e^{\prime}$. Hence by (19), $[c a]=0$ for all $a$. This proves the following theorem.

THEOREM 4. Let $\mathfrak{A}=\Phi_{n}$ the ring of $n \times n$ matrices over a field of characteristic 0 and let $\mathfrak{U}$ be a simple Jordan subalgebra of $\mathfrak{A}_{j}$. Then the center of $\mathfrak{U}$ coincides with the totality of elements $c$ of $\mathfrak{U}$ that commute in the ordinary multiplication with every $a$ in $\mathfrak{H}$.

The Johns Hopkins University 